\title{
SLC27A2 regulates miR-411 to affect chemo-resistance in ovarian cancer
}

\author{
F. D. CHEN, H. H. CHEN, S. C. KE, L. R. ZHENG, X. Y. ZHENG* \\ Department of Obstetrics and Gynecology, the Third Affiliated Hospital of Wenzhou Medical University, Wenzhou, China \\ *Correspondence: $z x y \_j e s s i c a @ 163 . c o m$
}

Received January 22, 2018 / Accepted May 2, 2018

\begin{abstract}
Although platinum-based chemotherapies have long been used as standard treatment in ovarian cancer, cisplatin resistance is a major problem that restricts its use. Herein, we investigate the biological function of SLC27A2 and its underlying mechanisms in regulating chemo-resistance in ovarian cancer. The findings show that SLC27A2 down-regulation in primary ovarian cancer tissues correlates with chemo-resistance and poor patient survival in our patient cohort. Significantly, we demonstrate that up-regulation of SLC27A2 by lentivirus-mediated p-SLC27A2 sensitizes ovarian cancer cells to cisplatin in vitro and in vivo via apoptosis. Mechanistic investigation reveals that miR-411 is the most strikingly over-expressed gene in response to ectopic expression of SLC27A2, but under-expressed in recurrent ovarian cancer tissues. Lower miR-411 expression contributes to ovarian cancer chemo-resistance in vitro and in vivo. Furthermore, SLC27A2 directly binds specific sites in the miR-411 promoter region and promoter activity decreases after mutation of putative SLC27A2-binding sites. This indicates that SLC27A2 is required for the transcriptional induction of miR-411. The luciferase assays also confirm that miR-411 directly targets ABCG2 in ovarian cancer, and overall findings establish the SLC27A2-miR-411-ABCG2 pathway in the regulation of ovarian cancer chemo-resistance with potential therapeutic applications.
\end{abstract}

Key words: ovarian cancer, SLC27A2, miR-411, ABCG2, chemo-resistance

Ovarian cancer is a major cause of cancer death in women and the most lethal gynecological cancer [1]. Although incremental advances in chemotherapy over the past four decades have improved median survival, the cure rate is essentially unchanged; with the average 5-years survival rate only $40 \%$ [2]. An important barrier to achieving better outcomes for these patients is that the mechanisms of chemo-resistance in ovarian cancer need to be refined.

SLC27A2 is a transmembrane protein responsible for transporting long-chain and very long-chain fatty acids into cells and activating intracellular signaling pathways. Others include protein kinase $\mathrm{C}$ and peroxisome proliferator-activated receptors which are members of the SLC27A solute carrier protein family and known fatty acid transporters (FATP) [3]. While SLC27A2 has a significantly deregulated gene signature in breast and lung cancer [4, $5]$, the underlying mechanisms and clinic correlation of SLC27A2 in ovarian cancer require further investigation. No studies on the biological function of SLC27A2 in regulating chemo-resistance in ovarian cancer appear in the literature.

MicroRNAs (miRNAs) are small, non-coding RNAs with approximately 19-24 nucleotides which regulate the expression of multiple-targeted genes by inducing translational silencing or causing degradation of the targets when combined with the RNA-induced silencing complex (RISC) [6]. Although current studies confirm that miRNA's have important roles in tumor suppression or promotion [7], the biological function and underlying mechanisms of a number of miRNAs are unclear and there is especial lack of studies on upstream miRNAs regulators.

Herein, we investigate the biological function of SLC27A2 and its underlying mechanisms in regulating chemo-resistance in ovarian cancer. We found that SLC27A2 downregulation in our patient cohort primary ovarian cancer tissues correlated with chemo-resistance and poor patient survival. In vitro and in vivo ovarian cancer cells were sensitized to cisplatin by up-regulation of SLC27A2 by lentivirusmediated p-SLC27A2. We demonstrated that SLC27A2 can directly bind to the specific site in the miR-411 promoter region and that ABCG2 was miR-411's target gene in ovarian cancer. The in vitro and in vivo assays established that downregulated miR-411 expression contributes to ovarian cancer chemo-resistance, and overall findings determined that SLC27A regulates miR-411 to effect chemo-resistance in ovarian cancer. 


\section{Materials and methods}

Animal and cell culture. Three-to four week-old female athymic BALB/c nu/nu mice obtained from HFK Bioscience (China) were maintained at the Animal Core Facility at the Third Affiliated Hospital of Wenzhou Medical University under specific pathogen-free (SPF) condition. All studies on mice were conducted in accordance with the National Institutes of Health 'Guide for the Care and Use of Laboratory Animals' and were approved by the ethical committee of the Third Affiliated Hospital of Wenzhou Medical University. SKOV3 and OVCAR3 ovarian cancer cell lines were purchased from ATCC. The identities of all cell lines were independently authenticated by short tandem repeat genotyping before the experiments. All cells were cultured according to ATCC recommendations.

Patient samples. 10 patients with primary ovarian cancer who consecutively underwent chemotherapy at the Third Affiliated Hospital of Wenzhou Medical University were enrolled in this study from January 2009 to June 2015. Informed consent for the additional core-needle biopsy and experimental use of tumor samples was obtained from all patients; following the protocol approved by the Ethics Committee of the Third Affiliated Hospital of Wenzhou Medical University. The tumor response was evaluated by the Response Evaluation Criteria in Solid Tumors (RECIST).

Lentiviral infection. SKOV3 and OVCAR3 dissociated were spin-infected with $1 \mathrm{ml}$ of NC-si-RNA (Shanghai GenePharma Co. Ltd, China), p-SLC27A2 lentiviral overexpression, or SLC27A2-si-RNA lentiviral knockdowns (Shanghai GenePharma Co. Ltd, China) packbag (the sequence for si-SLC27A2: 5'-CTCCCTGGATATTCTTAGTTT-3'). For both systems, cells were infected with lentiviral media at a multiplicity of infection (MOI) of 40, in the presence of $8 \mathrm{mg} / \mathrm{mL}$ polybrene (Sigma-Aldrich, USA), overnight in a $37^{\circ} \mathrm{C}$ incubator. Stable cell strains were constructed. The transduction efficiency was evaluated by GFP expression and stable clones were selected by puromycin.

Western blot analysis. Cellular proteins were harvested and equal amounts of protein were subjected to SDS-PAGE. Proteins were transferred to nitrocellulose membranes and the blots were probed with different primary antibodies, and developed by enhanced chemiluminescence reagents as previously described [8]. Antibody was purchased from Cell Signaling Technology: GAPDH (\#5174), dilution (1:1000); ABCG2 (\#42078), dilution (1:1000).

Animal studies and bioluminescence. All procedures were according to animal protocol. This was approved for the age-matched female mice. $3 \times 10^{6}$ luciferase-expressing ovarian cells were intraperitoneally injected, and the mice were separated into 4 treatment groups. In the DDP treatment group, a rodent diet with $625 \mathrm{mg} / \mathrm{kg}$ DDP (HarlanTeklad) was administered 14 days after the injection. We injected BALB/C nude mice subcutaneously with ovarian cancer Luc-cell-line cells established in our laboratory. Three mice in each group were injected. When the tumors reached approximately $5 \mathrm{~mm}$ in size, the LNA (Lock Nucleic Acid) decorated lenti-miR-411 was injected into the left side at $110 \mu \mathrm{g} / \mathrm{kg} /$ day and anti-nc was injected into right side at the same dosage. Tumor progression was monitored by bioluminescence (IVIS Spectrum), and survival was monitored daily. Tissue samples were collected on the indicated days after injection for pathological analysis.

Luciferase assays. Luciferase reporter assays were by Luciferase Assay System (Promega). The reporter gene construct and pTK-Renilla construct were co-transfected into cells, and luciferase activity was measured with the Dual-Luciferase Reporter Assay System (Promega). The results are expressed as luciferase/renilla ratios and the give average \pm standard deviation of at least three experiments, each performed in triplicate.

TaqMan qPCR array for chemo-resistance miRNA. TaqMan array plates of 80 miRNA for chemo-resistance and 4 assays for candidate endogenous control were used to perform quantitative PCR analysis (Applied Biosystems). A total of 6 cards were used to quantify mRNA expression. Each card was designed to run 2 distinct samples in triplicate and contained 384 wells and 8 reservoirs in total; thus providing 192 wells per sample in tripled 64 assays. $400 \mathrm{ng}$ of total RNA reverse transcribed in cDNA was used per sample (100 ng RNA/reservoir) in the TLDA cards. qPCR was performed on the Applied Biosystems ViiA ${ }^{\mathrm{Tm}} 7$ qPCR System using the following cycling conditions: $95 \mathrm{C}$ for 20 s, followed by 40 cycles of $95^{\circ} \mathrm{C}$ for 1 s and $60^{\circ} \mathrm{C}$ for 20 s. Cycle thresholds (CTs) were assessed as the average of each sample assayed in triplicate by Thermo Fisher Cloud online software (Life Technologies). The custom pre-amplification primer pool was also purchased from Thermo Fisher (\#4478705). Results were expressed as fold change, by comparing SLC27A2 over-expression by p-SLC27A2 with scrambled control after correction for housekeeping genes and utilizing the threshold cycle $(\mathrm{Ct})$ method and the $2^{\Delta \Delta \mathrm{Ct}}$ formula.

Quantitative RT-PCR (qPCR). Real-time PCR was performed by Applied Biosystems ${ }^{\bowtie}$ StepOne $^{\mathrm{TM}}$ Real-Time PCR Systems (Applied Biosystems). All qPCR reactions were performed in duplicate and the amplification signal from the target gene was normalized to a GAPDH signal. The TaqMan MicroRNA assay kit (Applied Biosystems) detected mature miR-25 and all qPCR reactions were performed in duplicate. The amplification signal from the target miRNA was normalized to a U6 signal, and herein we present the average of three experiments each performed in triplicate with standard error

Statistical analysis. Unless otherwise specified, all results are expressed as mean \pm SEM and analyzed by SPSS 20.0 software (SPSS, Chicago, IL, USA). Differences between groups were assessed using the Student's t-test. The relationship between SLC27A2 and miR-411 expression and the clinical-pathological features of ovarian cancer were analyzed by Pearson's $\chi^{2}$ test. The overall survival of the two patient 
groups was analyzed by the log-rank test using the KaplanMeier method, with $\mathrm{p}<0.05$ statistically significant.

\section{Results}

SLC27A2 down-regulation in primary ovarian cancer tissues correlates with chemo-resistance and poor patient survival. We compared SLC27A2 expression in 10 paired primary and recurrent tumor tissues to investigate if reduced expression of SLC27A2 is associated with chemo-resistance in the ovarian cancer patient cohort. Significantly decreased SLC27A2 levels were found in recurrent tumors (Figure 1A, Supplementary Table S1). While SLC27A2 down-regulation was significantly associated with reduced progression-free time (Figure 1B), the ovarian cancer patients were divided into groups which exhibited CR (chemo-sensitive), PR (chemo-sensitive), or PD (chemo-resistant) according to
RECIST criteria. SLC27A2 expression in PD ovarian tumors was the lowest in all chemo-responsive tissues (Figure 1C). This implies a potential role for SLC27A2 in regulating chemo-resistance which is widely believed to contribute to tumor relapse. We also determined that other compared ovarian cancer line cells, including Hey, A2780, Caov3, ES2 and Caov4, SKOV3 and OVCAR3 expressed relatively lower SLC27A2 level (Figure 1D), so we used SKOV3 and OVCAR3 for further study.

Ectopic expression of SLC27A2 sensitizes ovarian cancer cells to cisplatin. Lentivirus mediated p-SLC27A2 then detected if lower SLC27A2 expression in ovarian cancer cells is responsible for chemo-resistance. Efficiency of p-SLC27A2 and how si-SLC27A2 reversed SLC27A2 over-expression were determined by qPCR (Figures $2 \mathrm{~A}$ and B). Without the presence of cisplatin (DDP), cell viability was almost unchanged on infection with $\mathrm{p}-S L C 27 A 2$ or
A

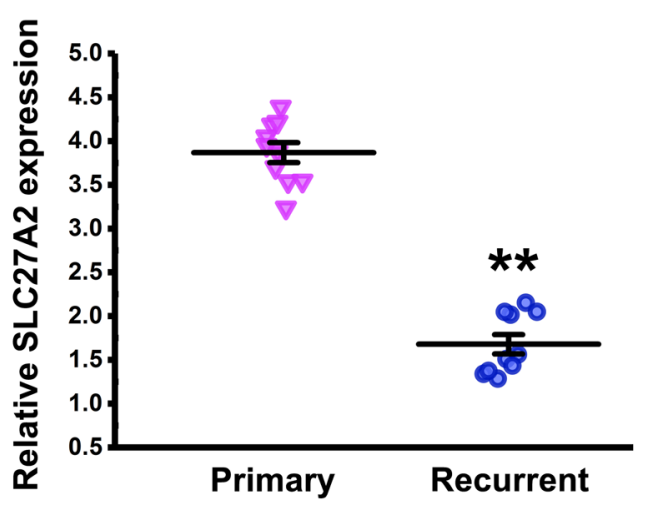

$\mathbf{B}$

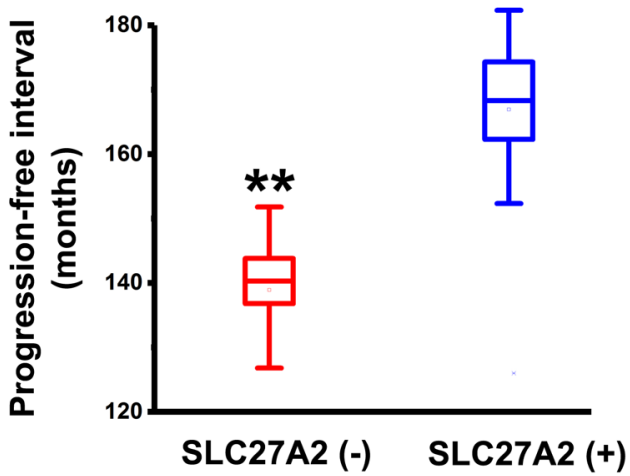

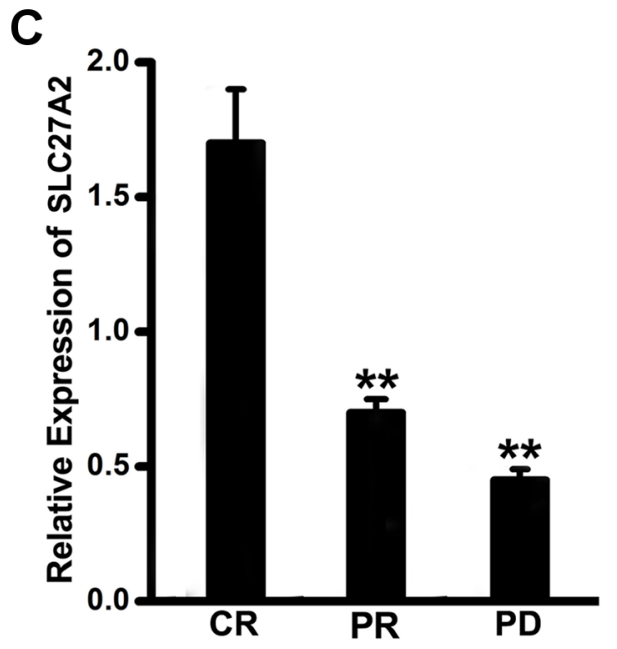

D

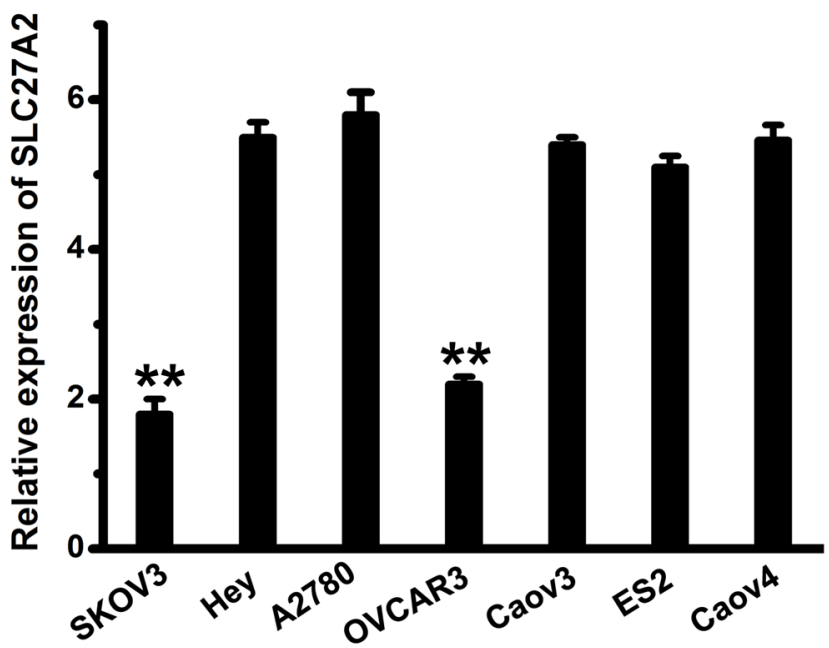

Figure 1. SLC27A2 down-regulation in primary ovarian cancer tissues correlates chemo-resistance. A) Representative expression of SLC27A2 in paired primary and recurrent ovarian cancer tissues. B) Comparison of the disease-free intervals in ovarian cancer patients with or without SLC27A2. C) Relative expression of SLC27A2 in different chemo-responsive ovarian cancer samples (CR: disappearance of the disease; PR: reduction of >30\%; PD: enlargement $>\mathbf{2 0} \%$ ) were examined by qPCR. D) Relative expression of SLC27A2 in different ovarian cancer cell lines, including Hey, A2780, Caov3, ES2, Caov4, SKOV3 and OVCAR3. Note: Columns, mean of three individual experiments; SD, ${ }^{* *} \mathrm{p}<0.01$. 

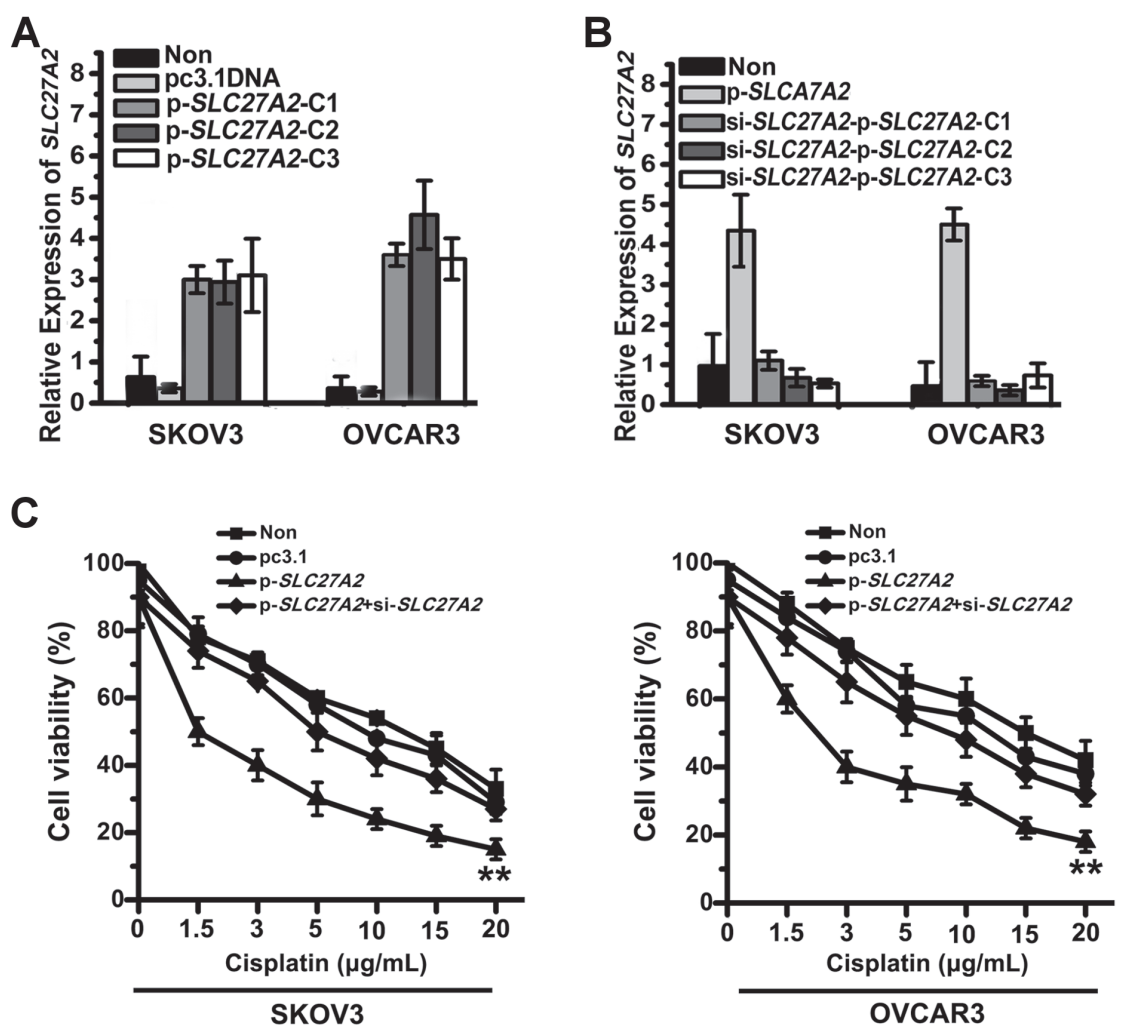

D

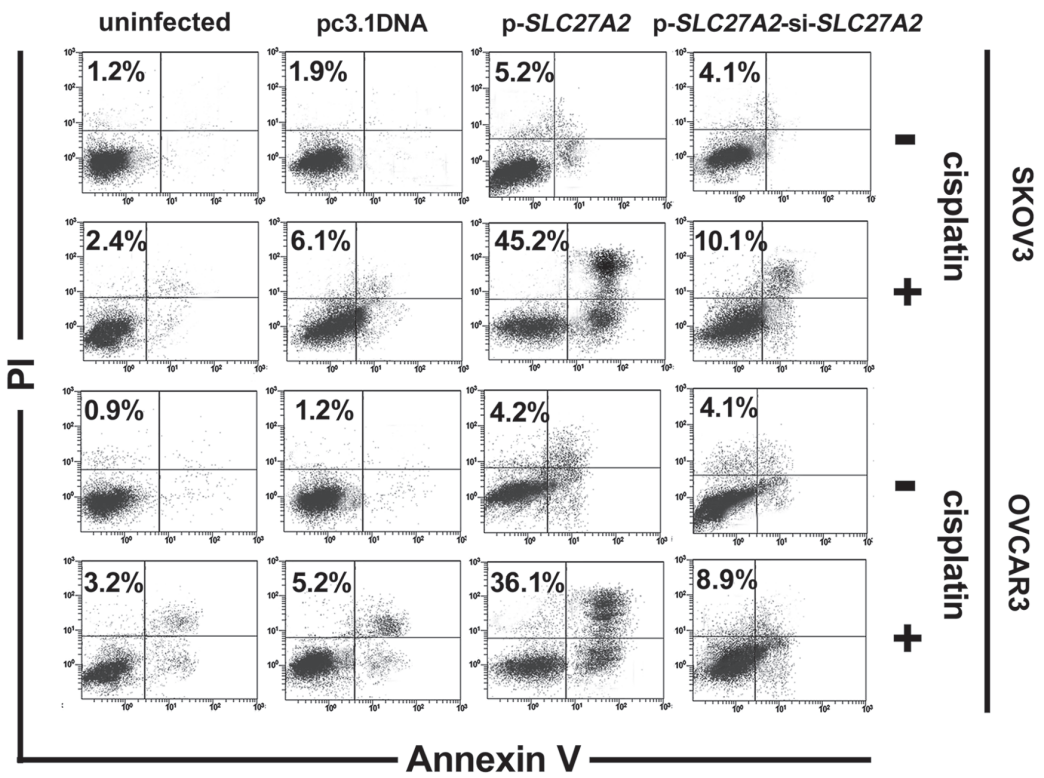

Figure 2. Ectopic expression of SLC27A2 sensitizes ovarian cancer cells to cisplatin via apoptosis. A) qPCR analysis was performed for selection of lenti-p-SLC27A2 stably expressed cell lines. B) qPCR analysis was performed for selection of lenti-p-SLC27A2-si-SLC27A2 stably expressed cell lines. C) The population of viable ovarian cancer cells (uninfected) and the population of viable cells upon infection with lenti-p-SLC27A2, lenti-pc3.1DNA or co-infection with lenti-si-SLC27A2 and lenti-p-SLC27A2 were measured by MTT when they were all treated with increasing concentrations of cisplatin from 40 to $240 \mathrm{mM}$. Note: Columns, mean of three individual experiments; SD, ${ }^{* *} \mathbf{p}<0.01$. D) With or without cisplatin, the percentage of Annexin $\mathrm{V}^{+} / \mathrm{PI}^{+} /$Annexin $\mathrm{V}^{+} / \mathrm{PI}^{-}$which was enhanced by ectopic expression of SLC27A2 was measured. co-infection with si-SLC27A2 and p-SLC27A2; thus suggesting that down-regulated SLC27A2 expression was not required for ovarian cancer cell survival and self-renewal. When ovarian cancer cells were treated with increasing concentrations of DDP, cell viability decreased more rapidly in the p-SLC27A2 group than in the uninfected group and the pc3.1DNA control group (Figure 2C). Reversing SLC27A2 expression by infection with si-SLC27A2 in p-SLC27A2 group increased cell viability in the presence of DDP (Figure 2C). The Annexin V/PI staining assay then showed that infection with $\mathrm{p}-S L C 27 A 2$ increased the percentage of Annexin $\mathrm{V}^{+} / \mathrm{PI}^{+} /$Annexin $\mathrm{V}^{+} / \mathrm{PI}^{-}$cells compared to uninfected cells and the pc3.1DNA control group (Figure 2D). This demonstrates that up-regulated SLC27A2 expression level in ovarian cancer cells relieves chemoresistance to DDP by apoptosis.

Combination of SLC27A2 overexpression and chemo-therapy suppresses in vivo tumor growth. Figure $3 \mathrm{~A}$ depicts the treatment of mice bearing i.p. xenografts with p-SLC27A2 stable cell line cells, DDP and the paclitaxel (PTX) we used to test the combined effect of SLC27A2 inhibition and chemotherapy. Three mice were injected in each group and intra-peritoneal implantation of p-SLC27A2 stable cell line cells led to marginal in vivo tumor reduction (Figure 3B). The combination of the two treatments and p-SLC27A2 stable cell line cells significantly suppressed tumor growth (Figure 3B). Moreover, the combined p-SLC27A2 stable cell line cells, PTX and DDP treatment prolonged mice survival (Figure $3 \mathrm{C})$ and these results suggest that ectopic expression of SLC27A2 can be combined with current standard-ofcare chemotherapy to provide therapeutic blocking of progression.

miR-411 is down-regulated in ovarian cancer and contributes to SLC27A2 mediated chemo-resistance. Quantitative PCR analysis assessed the underlying mechanisms of SLC27A2 on chemo-resistance in 


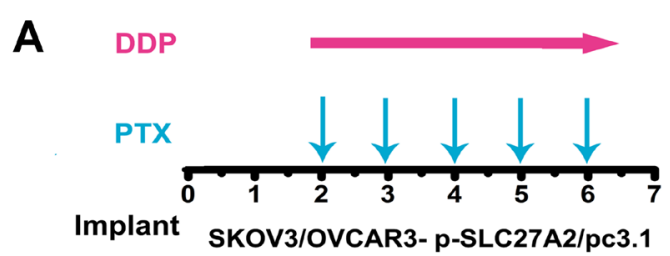

B

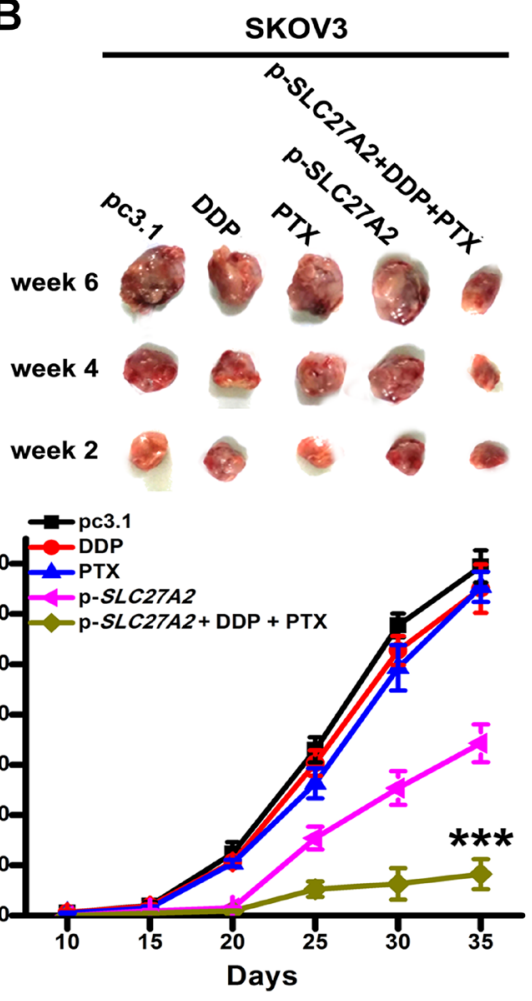

C

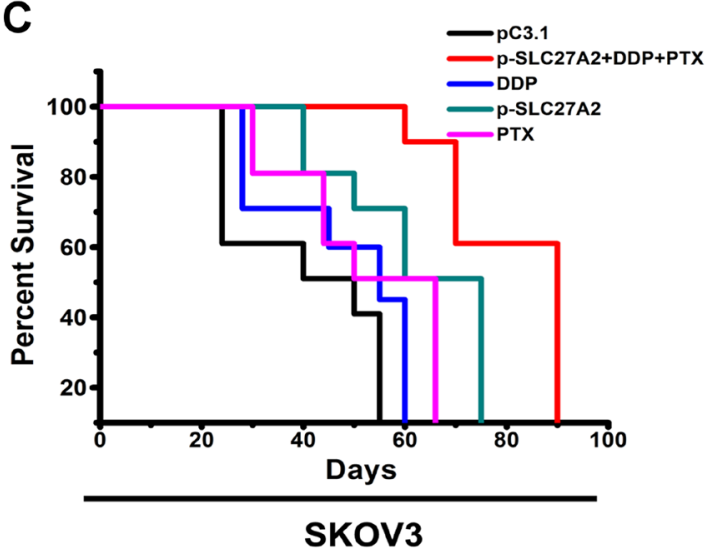

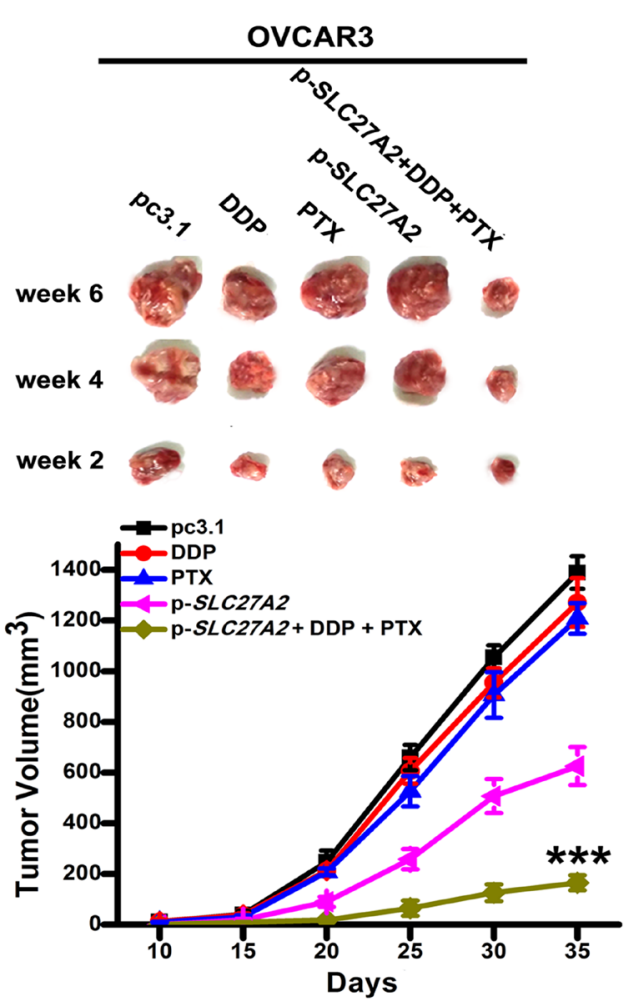

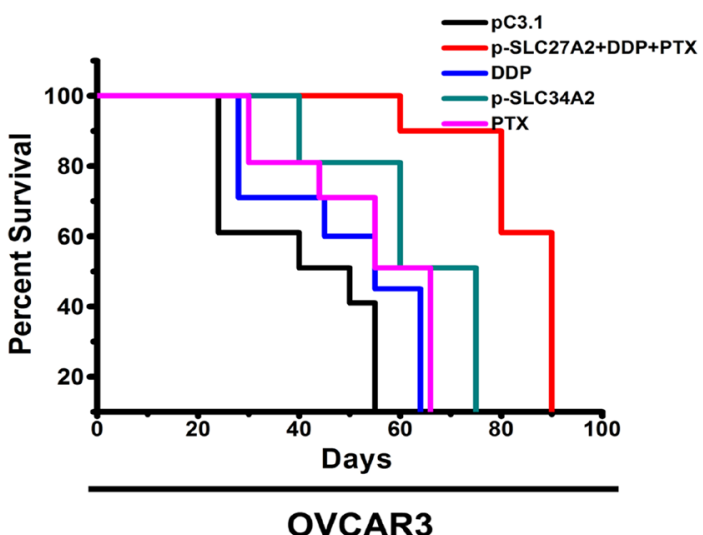

Figure 3. Combined SLC27A2 over-expression and chemo-therapy suppresses tumor growth in vivo. A) Mice were separated into 5 treatment groups according to the schema depicted. B) Weekly representative tumor images and curves of animals in each group are shown depicting tumor burden. Note: Columns, mean of three individual experiments; $\mathrm{SD},{ }^{\star *} \mathrm{p}<0.01$. C) Significant differences of survival between groups were determined by a logrank test.

ovarian cancer. Here, TaqMan ${ }^{\circledR}$ low-density array evaluated the differential expression of miRNAs associated with chemo-resistance. Figure $4 \mathrm{~A}$ shows that miR-411 is the most obviously up-regulated miRNA in response to p-SLC27A2 infection in ovarian cancer cells. The qPCR assay further verified the top 5 miRNAs (Figure $4 \mathrm{~B}$ ). 
We also established that the expression of miR-411 was decreased in recurrent tumors compared to their primary counterparts (Figure 4C). Moreover, cell viability decreased more rapidly in thelenti-miR-411 group than in the uninfected and lenti-NC control groups when ovarian cancer cells were treated with increasing concentrations of DDP (Figure 4D). Infection with lenti-miR-411 in si-SLC27A2 group increased cell viability in the presence of DDP (Figure 4D).

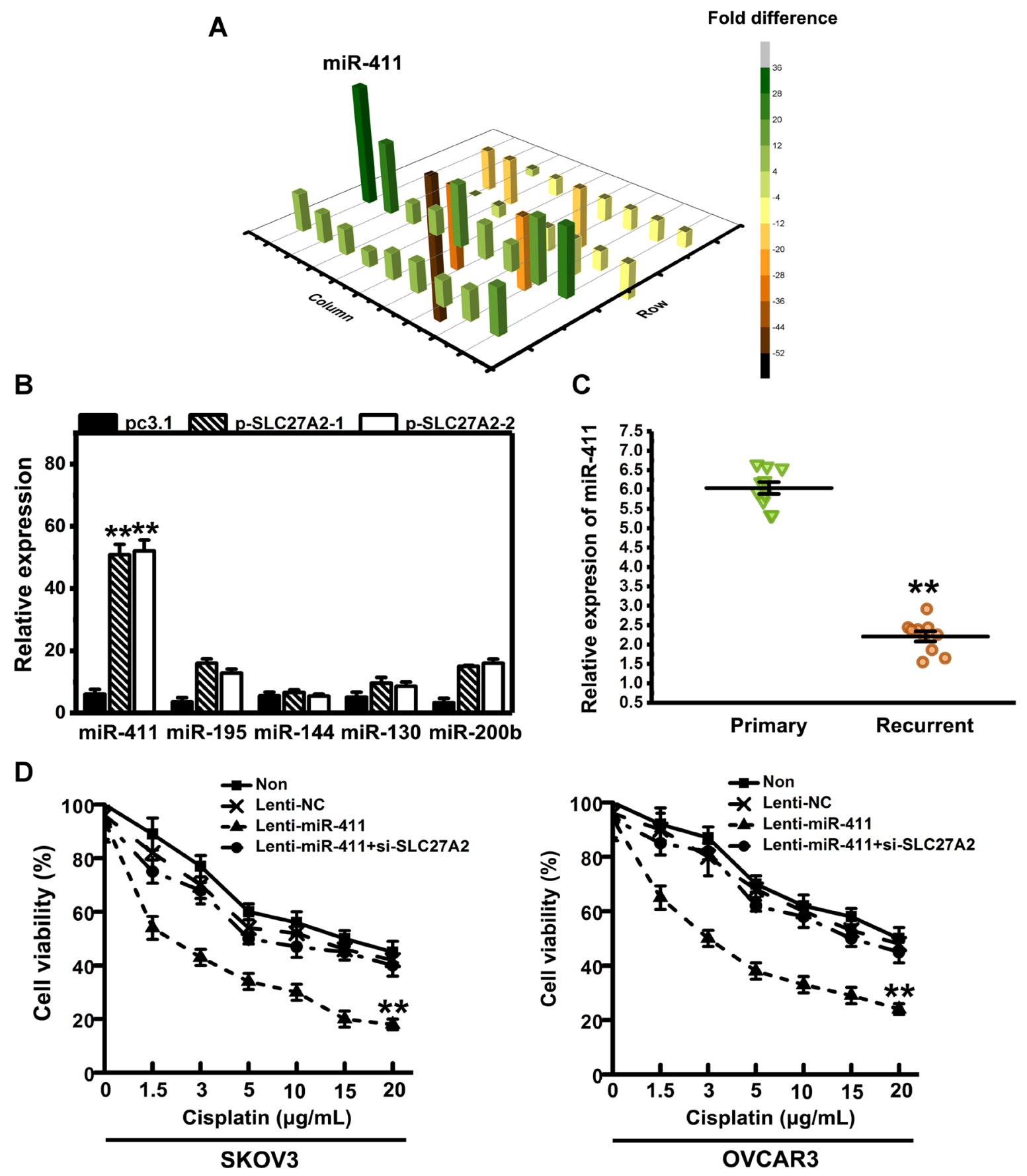

Figure 4. miR-411 is down-regulated in ovarian cancer. A) The miRNAs associated with chemo-resistance expression levels are represented in the 3D bar chart. B) qPCR analysis of some genes involved in miRNAs associated with chemo-resistance in ovarian cancer cells. C) Representative expression of miR-411 in paired primary and recurrent ovarian cancer tissues. D) The population of viable ovarian cancer cells (uninfected) and the population of viable cells upon infection with lenti-miR-411, lenti-NC or co-infection with lenti-miR-411 and lenti-si-SLC27A2 were measured by MTT when they were all treated with increasing concentrations of cisplatin from 40 to $240 \mathrm{mM}$. Note: Columns, mean of three individual experiments; SD, ${ }^{* *} \mathrm{p}<0.01$. 
A

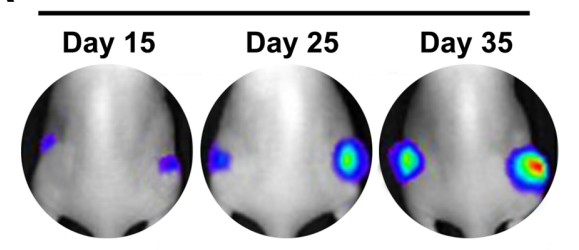

B

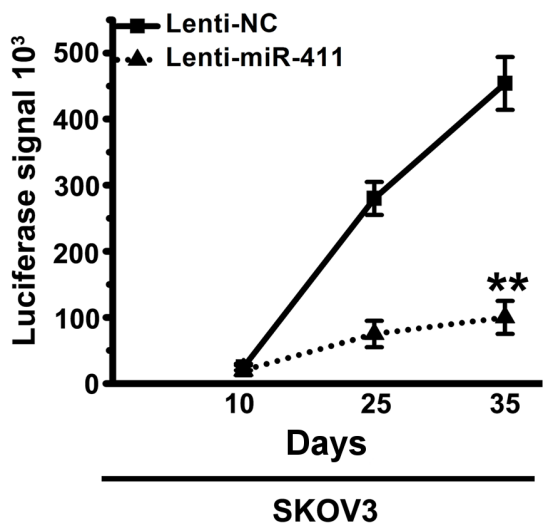

OVCAR3
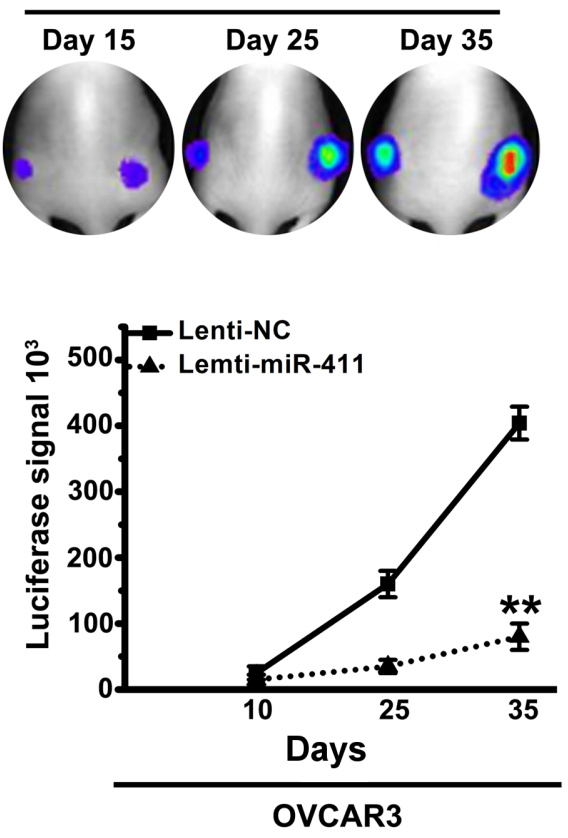

Figure 5. Ectopic expression of miR-411 modulates chemo-resistant in tumor-bearing nude mice. A) and B) Representative images showed the luciferase signals of tumors treated or untreated with miR-411 on the back of nude mice on the day 15, 25, and 35 under the treatment of cisplatin (A). Comparison of luciferase signals on the day 35 was analyzed and indicated (B). Note: Columns, mean of three individual experiments; $\mathrm{SD}$, ${ }^{\star *} \mathrm{p}<0.01$.

In vivo animal study. We subcutaneously injected BALB/C nude mice with ovarian cancer Luc-cell-line cells established in our laboratory to test in vivo relationships between miR-411 and cisplatin-resistance. Three mice were injected in each group. When the tumors reached approximately $5 \mathrm{~mm}$, the Lock Nucleic Acid decorated lenti-miR-411 was injected into the left side at $110 \mu \mathrm{g} / \mathrm{kg} / \mathrm{day}$ and lenti-nc was injected into right side in the same dosage (Figures 5A, 5B). The mice were intraperitoneally injected three days later with PBS and cisplatin chemotherapeutic drugs in two injections a week. The tumor luciferase signals were tested every week by IVIS 100 and the 15, 25 and 35 day results are in Figure 5B.

ABCG2 is the direct target of miR-411 which is regulated by SLC27A2 in ovarian cancer. A series of assays evaluated if SLC27A2 could control miR-411. There was initial significant increase in mature miR-411, pre-miR-411 and pri-miR-411 in the p-SLC27A2 group (Fig 6A). The promoter region containing numerous putative SLC27A2 binding sites was investigated by luciferase reporter assay to further confirm the transcriptional control of miR-411 by SLC27A2. This showed that over-expression or knockdown of SLC27A2 significantly enhanced or inhibited miR-411 promoter activity, respectively, thus suggesting that SLC27A2 directly binds specific sites in the miR-411 promoter region (Figure 6B).

We then applied computational target prediction to determine the ABCG2 gene as a potential target of miR-411 (Figure
6C). Luciferase activity of WT-ABCG2 3'UTR was significantly reduced by miR-411 in a dose-dependent manner, but no significant difference in MT-ABCG2 3'UTR luciferase activity was noted (Figure 6D). The miR-411 knockdown restored the p-SLC27A2-inhibited ABCG2 expression to a certain extent (Figure 6E). This implies that low SLC27A2 expression induces ABCG2 expression by miR-411 downregulation.

\section{Discussion}

Although incremental advances in chemotherapy over the past four decades have improved median survival, the cure rate in ovarian cancer is essentially unchanged [2]. The mechanisms of chemo-resistance in ovarian cancer should therefore be investigated. The SLC27A2 transmembrane protein is responsible for transporting long-chain and very long-chain fatty acids into the cell and activating intracellular signaling pathways [3]. Januchowski et al. [9] are the only researchers to report that SLC27A2 was significantly downregulated in chemo-resistant variants of A2780 in ovarian cancer. However, they provided no indication of the underlying mechanisms and clinic correlation, and no known studies have investigated the biological function of SLC27A2 in regulating chemo-resistance in ovarian cancer.

Herein, we showed the significantly decreased 1 SLC27A2 level in recurrent tumors compared to their primary counterparts (Figure 1A). SLC27A2 down-regulation was also 
A

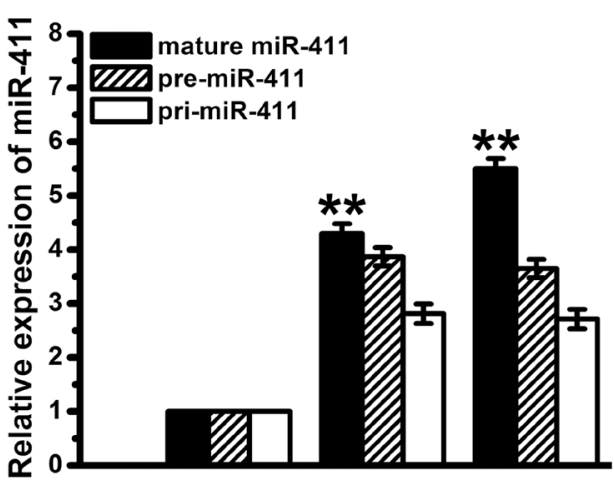

p-SLC27A2 nc

\section{B}

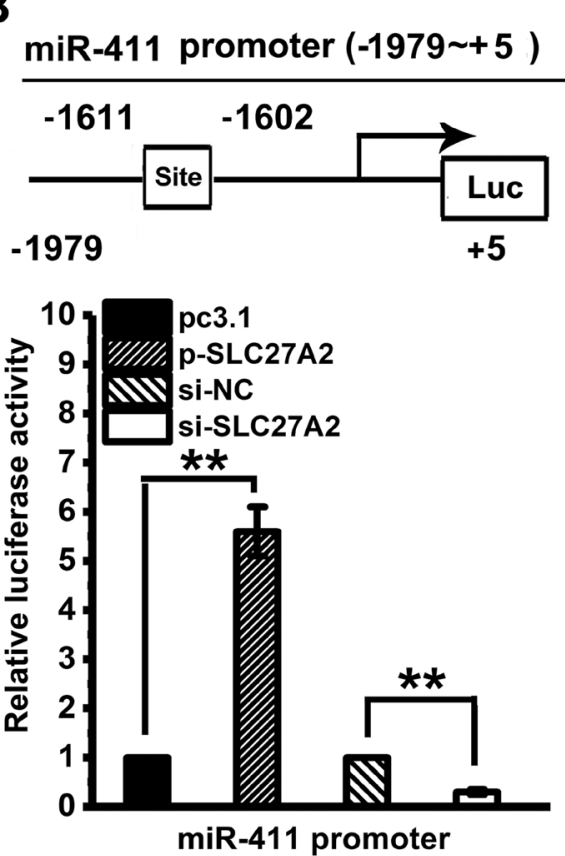

E

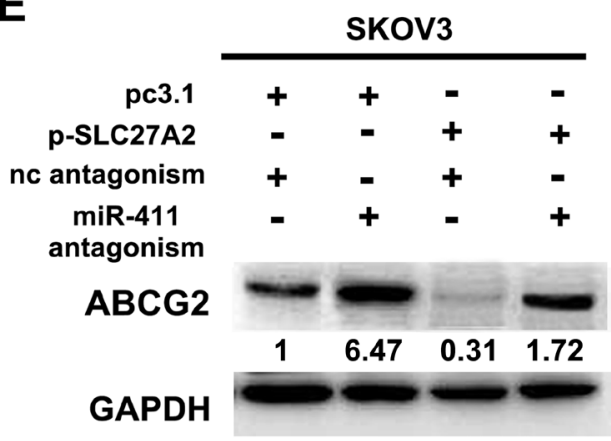

\#2
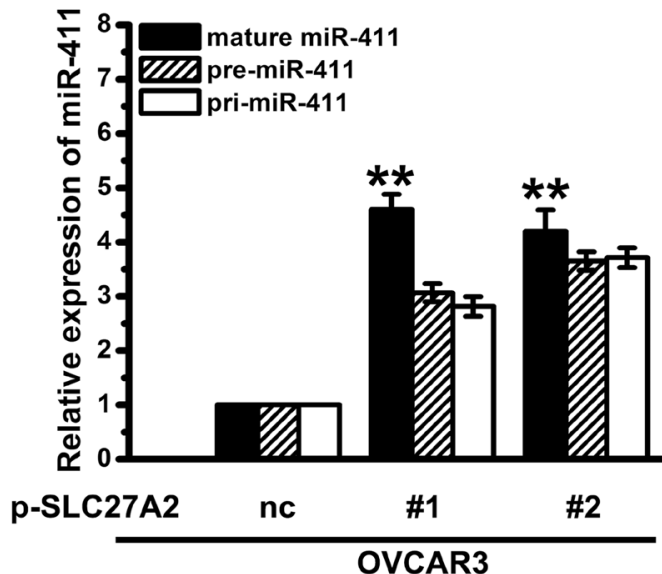

C

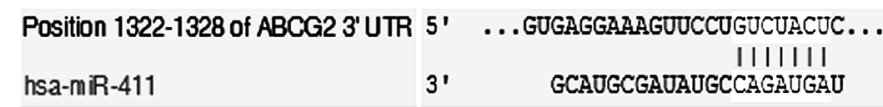

D
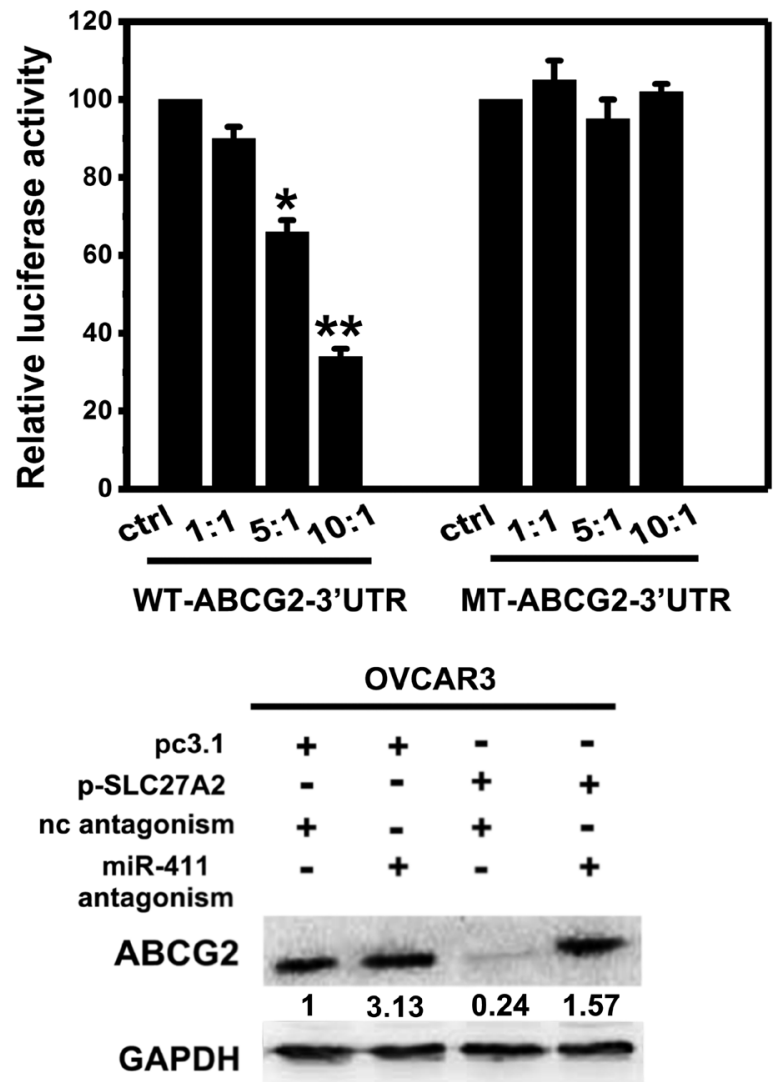

Figure 6. Low expression level of SLC27A2 induced ABCG2 expression via the down-regulation of miR-411. A) The expression of mature miR-411, pre-miR-411, and pri-miR-411 was detected in ovarian cancer cells by qPCR. B) Luciferase reporter assays were carried out using SKOV3 cells by overexpressing SLC27A2 (p-SLC27A2) or the vector controls or cells expressing either a control si-NC or a si-SLC27A2. C) The diagram shows miR-411 and its putative binding site at the 3'-UTR of ABCG2 and the sequences of WT and MT ABCG2 3'-UTR using SKOV3 cells. D) A luciferase reporter assay was used to assess whether miR-411 can directly bind to the 3 '-UTR of ABCG2. Note: Columns, mean of three individual experiments; SD, ${ }^{\star *}$ p $<0.01$. E) Western-blot analysis was used to detect the protein expression of ABCG2 where SLC27A2 was ectopic expression or miR-411 was knocked down. GAPDH was used as a loading control. 
found significantly associated with reduced progression-free interval and had the lowest expression in PD ovarian tumors (Figures 1B, C). This suggests the potential role of SLC27A2 in regulating chemo-resistance which is widely believed to contribute to tumor relapse. We also found that SKOV3 and OVCAR3 expressed relatively lower levels of SLC27A2 than other ovarian cancer line cells, including Hey, A2780, Caov3, ES2 and Caov4, (Figure 1C). The reason may be that SKOV3 and OVCAR3 cell lines are DDP resistant, and this suggests that SLC27A2 is most likely associated with DDP resistance.

To detect whether lower SLC27A2 expression in ovarian cancer cells is responsible to the chemo-resistance, we showed that when ovarian cancer cells were treated with increasing concentrations of DDP, cell viability decreased more rapidly in p-SLC27A2 group. Reversing SLC27A2 expression by infection with si-SLC27A2 in the p-SLC27A2 group increased cell viability in the presence of DDP (Figure 2B). Annexin V/PI staining assay then showed that infection with $\mathrm{p}-S L C 27 A 2$ increased the percentage of Annexin $\mathrm{V}^{+} / \mathrm{PI}^{+} /$Annexin $\mathrm{V}^{+} / \mathrm{PI}^{-}$cells (Figure 2C). This demonstrates that up-regulated SLC27A2 expression level in ovarian cancer cells relieves chemo-resistance to DDP by apoptosis. Although p-SLC27A2 intraperitoneal implantation led to marginal tumor reduction in vivo, the combination of the two treatments (PTX and DDP) and p-SLC27A2 suppressed tumor growth significantly and prolonged mice survival (Figure 3). This suggests that SLC27A2 inhibition can be combined with current standard-of-care chemotherapy to provide successful blocking of cancer progression.

TaqMan ${ }^{\circledast}$ low-density array investigated involved mechanisms and revealed that miR-411 was the most obviously up-regulated miRNA in response to p-SLC27A2 infection in ovarian cancer cells (Figure 4A).

Previously, miR-411 has been reported a potential tumorsuppressor-miRNA which can inhibit proliferation and metastasis in breast cancer by targeting different genes [10, 11], but there is no evidence of it providing chemo-resistance in cancer. In ovarian cancer, There has been only the one paper by Kim YW et al. relating miR-411 to ovarian cancer, and these authors reported that miR-411 was under expressed in Taxol resistant ovarian cancer cells [12]. However, its exact biological function and the underlying mechanisms remain unknown.

Herein, we confirm that miR-411 expression was decreased in recurrent tumors compared to their primary counterparts, and that down-regulated miR-411 in ovarian cancer contributed to SLC27A2 mediated chemo-resistance in vitro and in vivo (Figure 4 and 5). Luciferase reporter assay then showed that over-expression or knockdown of SLC27A2 significantly enhanced or inhibited miR-411 promoter activity, respectively, thus suggesting that SLC27A2 directly binds specific sites in the miR-411 promoter region (Figure 6B).

We then established that the ABCG2 gene was the direct target of miR-411 (Figure 6C). This implies that low SLC27A2 expression level induces ABCG2 expression by down-regulating miR-411. The ABC transporter family has at least 48 members identified in humans, and 12 of these are recognized putative drug transporters [13-15]. When tumor patients have higher expression of $\mathrm{ABC}$ transporter pumps, they usually do not respond to chemotherapy because the $\mathrm{ABC}$ transporters located on the cytoplasm side of the resistant cell effluxes chemotherapeutic drugs out of the tumor cells, thus leading to chemo-resistance [16]. ABCG2 has previously been reported to be associated with chemo-resistance in different types of cancer $[5,17]$. Although ovarian cancer studies report that higher ABCG2 expression levels contribute to cisplatin chemo-resistance, ABCG2 upstream regulation employs different mechanisms [17-19], so we focused on its upstream regulation .

In conclusion, we established that SLC27A2 downregulation in primary ovarian cancer tissues correlates with chemo-resistance and poor patient survival. Our findings confirm that the SLC27A2-miR-411-ABCG2 pathway regulates ovarian cancer chemo-resistance and that blocking the progression of this pathway should provide therapeutic applications for this cancer.

Supplementary information is available in the online version of the paper.

\section{References}

[1] SIEGEL R, NAISHADHAM D, JEMAL A. Cancer statistics, 2013. CA Cancer J Clin 2013; 63: 11-30. https://doi. org/10.3322/caac. 21166

[2] BARNHOLTZ-SLOAN JS, SCHWARTZ AG, QURESHI F, JACQUES S, MALONE J et al. Ovarian cancer: changes in patterns at diagnosis and relative survival over the last three decades. Am J Obstet Gynecol 2003; 189: 1120-1127. https:// doi.org/10.1067/S0002-9378(03)00579-9

[3] STAHL A. A current review of fatty acid transport proteins (SLC27). Pflugers Arch 2004; 447: 722-727. https://doi. org/10.1007/s00424-003-1106-Z

[4] SHUBBAR E, KOVACS A, HAJIZADEH S, PARRIS TZ, NEMES $S$ et al. Elevated cyclin B2 expression in invasive breast carcinoma is associated with unfavorable clinical outcome. BMC Cancer 2013; 13: 1. https://doi.org/10.1186/14712407-13-1

[5] SU J, WU S, TANG W, QIAN H, ZHOU H et al., Reduced SLC27A2 induces cisplatin resistance in lung cancer stem cells by negatively regulating Bmil-ABCG2 signaling. Mol Carcinog 2016; 55: 1822-1832. https://doi.org/10.1002/mc.22430

[6] BARTEL DP. MicroRNAs: genomics, biogenesis, mechanism, and function. Cell 2004; 116: 281-297. https://doi. org/10.1016/S0092-8674(04)00045-5

[7] BARTEL DP. MicroRNAs: target recognition and regulatory functions. Cell 2009; 136: 215-233. https://doi.org/10.1016/j. cell.2009.01.002

[8] SU J, WU S, WU H, LI L, GUO T. CD44 is functionally crucial for driving lung cancer stem cells metastasis through Wnt/beta-catenin-FoxM1-Twist signaling. Mol Carcinog 2016; 55: 1962-1973. https://doi.org/10.1002/mc.22443 
[9] JANUCHOWSKI R, ZAWIERUCHA P, RUCINSKI M, ANDRZEJEWSKA M, WOJTOWICZ K et al. Drug transporter expression profiling in chemoresistant variants of the A2780 ovarian cancer cell line. Biomed Pharmacother 2014; 68: 447-453. https://doi.org/10.1016/j.biopha.2014.02.002

[10] GUO L, YUAN J, XIE N, WU H, CHEN W et al. miRNA-411 acts as a potential tumor suppressor miRNA via the downregulation of specificity protein 1 in breast cancer. Mol Med Rep 2016; 14: 2975-2982. https://doi.org/10.3892/ mmr.2016.5645

[11] ZHANG Y, XU G, LIU G, YE Y, ZHANG C et al. miR-411$5 p$ inhibits proliferation and metastasis of breast cancer cell via targeting GRB2. Biochem Biophys Res Commun 2016; 476: 607-613. https://doi.org/10.1016/j.bbrc.2016.06.006

[12] KIM YW, KIM EY, JEON D, LIU JL, KIM HS et al. Differential microRNA expression signatures and cell typespecific association with Taxol resistance in ovarian cancer cells. Drug Des Devel Ther 2014; 8: 293-314. https://doi. org/10.2147/DDDT.S51969

[13] GILLET JP, EFFERTH T, REMACLE J. Chemotherapy-induced resistance by ATP-binding cassette transporter genes. Biochim Biophys Acta 2007; 1775: 237-262. https://doi. org/10.1016/j.bbcan.2007.05.002
[14] HOU H, KANG Y, LI Y, ZENG Y, DING G et al. miR33a expression sensitizes Lgr5+ HCC-CSCs to doxorubicin via ABCA1. Neoplasma 2017; 64: 81-91. https://doi. org/10.4149/neo_2017_110

[15] ZHANG L, GUO X, ZHANG D, FAN Y, QIN L et al. Upregulated miR-132 in Lgr5+ gastric cancer stem cell-like cells contribute to cisplatin-resistance via SIRT1/CREB/ABCG2 signaling pathway. Mol Carcinog 2017; 56: 2022-2034. https://doi.org/10.1002/mc.22656

[16] LAGE H. ABC-transporters: implications on drug resistance from microorganisms to human cancers. Int J Antimicrob Agents 2003; 22: 188-199. https://doi.org/10.1016/S09248579(03)00203-6

[17] CIOFFI M, D’ALTERIO C, CAMERLINGO R, TIRINO V, CONSALES $\mathrm{C}$ et al. Identification of a distinct population of CD133(+)CXCR4(+) cancer stem cells in ovarian cancer. Sci Rep 2015; 5: 10357. https://doi.org/10.1038/srep10357

[18] CHEN Y, BIEBER MM, TENG NN. Hedgehog signaling regulates drug sensitivity by targeting $\mathrm{ABC}$ transporters $\mathrm{ABCB} 1$ and ABCG2 in epithelial ovarian cancer. Mol Carcinog 2014; 53: 625-634. https://doi.org/10.1002/mc.22015

[19] XIONG X, ARVIZO RR, SAHA S, ROBERTSON DJ, MCMEEKIN S et al. Sensitization of ovarian cancer cells to cisplatin by gold nanoparticles. Oncotarget 2014; 5: 6453-6465. https://doi.org/10.18632/oncotarget.2203 


\section{SLC27A2 regulates miR-411 to affect chemo-resistance in ovarian cancer}

F. D. CHEN, H. H. CHEN, S. C. KE, L. R. ZHENG, X. Y. ZHENG*

\section{Supplemental Material}

Table S1. Expression of SLC27A2 in ovarian cancer samples.

\begin{tabular}{|c|c|c|c|c|c|c|}
\hline Case & Age & Gender & TNM & $\begin{array}{c}\text { Clinical } \\
\text { stage }\end{array}$ & $\begin{array}{c}{ }^{\text {a Normalized } S L C 27 A 2} \\
\text { amount } 2^{-\Delta \Delta \mathrm{Ct}}\end{array}$ & $\begin{array}{c}\text { Primary or } \\
\text { recurrent }\end{array}$ \\
\hline 1 & 41 & Female & T1N0M0 & IA & 3.5617 & primary \\
\hline 2 & 43 & Female & T2N0M0 & IB & 4.5011 & primary \\
\hline 3 & 68 & Female & T1N0M0 & IIA & 4.2456 & primary \\
\hline 4 & 66 & Female & T2N2M0 & IIB & 1.3918 & recurrent \\
\hline 5 & 75 & Female & T3N0M0 & IIIA & 3.7531 & primary \\
\hline 6 & 55 & Female & T2N3M0 & IIIB & 1.7974 & recurrent \\
\hline 7 & 68 & Female & $\mathrm{T} 2 \mathrm{~N} 3 \mathrm{M} 0$ & IIIB & 1.3732 & recurrent \\
\hline 8 & 77 & Female & T2N0M0 & IIIA & 4.0127 & primary \\
\hline 9 & 56 & Female & $\mathrm{T} 3 \mathrm{~N} 3 \mathrm{M} 1$ & IV & 1.1469 & recurrent \\
\hline 10 & 71 & Female & T3N3M1 & IV & 1.3337 & recurrent \\
\hline
\end{tabular}

${ }^{a}$ Relative quantification was performed by the $2^{-\Delta \Delta C t}$ method with the primary ovarian tissue samples as the calibrator. Data show the means from three independent analyses. Every independent analysis was carried out after the RNA extraction step. Total RNA was poly-A tailed, reverse transcript, and then real-time PCR tested. $\Delta \mathrm{C}_{\mathrm{T}}$ obtained from real-time PCR was subject to paired t-test $\left(\Delta \mathrm{C}_{\mathrm{T}}=\mathrm{C}_{\mathrm{T} \mathrm{SLC27A2}}-\mathrm{C}_{\beta-\text {-actin }}\right)$. The expression levels of SLC27A2 in recurrent ovarian cancer tissues were significantly lower than primary ovarian cancer tissues $(p<0.01)$. 